\section{CR YSTALLIZATION.}

\section{$T$}

HERE is something very fascinating about crystals. It is not merely the intrinsic beauty of their forms, their picturesque grouping, and the play of light upon their faces, but there is a feeling of wonder at the power of Nature, which causes substances, in passing from the fluid to the solid state, to assume regular shapes bounded by plane faces, each substance with its own set of forms, and its faces arranged with characteristic symmetry: some, like alum, in perfect octahedra ; others, like blue vitriol, in shapes which are regularly oblique. It is this power of Nature which is the subject of this discourse. I hope to show that crystalline forms, with all their regularity and symmetry, are the outcome of the accepted principles of mechanics. I shall invoke no peculiar force, but only such as we are already familiar with in other facts of Nature. I shall call in only the same force that produces the rise of a liquid in a capillary tube and the surface-tension at the boundary of two substances which do not mix. Whether this force be different from gravity I need not stop to inquire, for any attractive force which for small masses, such as we suppose the molecules of matter to be, is only sensible at insensible distances is sufficient for my purpose.

We know that the external forms of crystals are intimately connected with their internal structure. This is betrayed by the cleavages with which in mica and selenite everybody is familiar, and which extend to the minutest parts, as is seen in the tiny rhombs which form the dust of crushed calcite. It is better marked by the optical properties, single and double refraction, and the effects of crystals on polarized light. . These familiar facts lead up to the thought that it is really the internal structure which determines the external form. As a starting-point for considering that structure, I assume that crystalline matter is made up of molecules, and that, whereas in the fluid state the molecules move about amongst themselves, in the solid state they have little freedom. They are always within the range of each other's influence, and do not change their relative places. Nevertheless, these molecules are in constant and very rapid motion. Not only will they communicate heat to colder bodies in contact with them, but they are always radiating, which means producing waves in the ether at the rate of many billions in a second. We are sure that they have a great deal of energy, and, if they cannot move far, they must have very rapid vibratory motions. It is reasonable to suppose that the parts of each molecule swing, backwards and forwards, through, or about, the centre of mass of the molecule. The average distances to which the parts swing will determine the average dimensions of the molecule, the average space it occupies.

Dalton fancied he had proved that the atoms of the chemical elements must be spherical, because there was no assignable cause why they should be longer in one dimension than another. I rather invert his argument. I see no reason why the excursions of the parts of a molecule from the centre of mass should be equal in all directions, and therefore assume, as the most general case, that these excursions are unequal in different directions. And, since the movements must be symmetrical with reference to the centre of mass of the molecule, they will in general be included within an ellipsoid, of which the centre is the centre of mass.

Here I may, perhaps, guard against a misconception. We chemists are familiar with the notion of complex molecules; and most of us figure to ourselves a molecule of common salt as consisting of an atom of sodium and one of chlorine held together by some sort of force, and it may be imagined that these atoms are the parts of

1 A Diccourse delivered at the Royal Institution of Great Britain on Friday, May 15, 1891, by G. D. Liveing, F.R.S. NO. I I 29 , VOL. 44.$]$ the molecules which I have in mind. That, however, is not my notion. I am paradoxical enough to disbelieve altogether in the existence of either sodium or chlorine in common salt. Were my audience a less philosophical one I could imagine I heard the retort from many a lip : "Why, you can get sodium and chlorine out of it, and you can make it out of sodium and chlorine!" But no, you cannot get either sodium or chlorine out of common salt without first adding something which seems to me of the essence of the matter. You can get neither sodium nor chlorine from it without adding energy; nor can you make it out of these elements without subtracting energy. My point is that energy is of the essence of the molecule. Each kind of molecule has its own motion; and in this I think most physicists will agree with me. Chemists will agree with me in thinking that all the molecules of the same element, or compound, are alike in mass, and in the space they occupy at a given temperature and pressure. The only remaining assumption I make is that the form of the ellipsoid--the relative lengths of its axes-is on the average the same for all the molecules of the same substance. This implies that the distances of the excursions of the parts of the molecule depend on its constitution, and are, on the average, the same in similarly constituted molecules under similar circumstances.

I have come to the end of my postulates. I hope they are such as you will readily concede. I want you to conceive of each molecule as having its parts in extremely rapid vibration, so that it occupies a larger space than it would occupy if its parts were at rest; and that the excursions of the parts about the centre of mass are on the average, at a given temperature and pressure, comprised within a certain ellipsoid; that the dimensions of this ellipsoid are the same for all molecules of the same chemical constitution, but different for molecules of different kinds.

We have now to consider how these molecules will pack themselves on passing from the fluid state, in which they can and do move about amongst themselves, into the solid state, in which they have no sensible freedom. If they attract one another, according to any law, and for my purpose gravity will suffice, then the laws of energy require that for stable equilibrium the potential energy of the system shall be a minimum. This is the same, in the case we are considering, as saying that the molecules shall be packed in such a way that the distances between their centres of mass shall on the whole be the least possible; or, that as many of them as possible shall be packed into unit space. In order to see how this packing will take place, it will be easiest to consider first the particular case in which the axes of the ellipsoids are all equal-that is, when the ellipsoids happen to be spheres. The problem is then reduced to finding how to pack the greatest number of equal spherical balls into a given space. It is easy to reduce this to the problem of finding. how the spheres can be arranged so that each one shall be touched by as many others as possible. In this way the cornered spaces between the balls, the unoccupied room, is reduced to a mirimum. You can stack balls so that each is touched by twelve others, but not by more. At first sight it seems as if this might be done in two. ways.

In the first place we may start with a square of balls, as in Fig. I, where each is touched by four others. We may then place another (shaded in the figure) so as to rest on four, and place four more in adjacent holes to touch it, as indicated by the dotted circles. Above these four more may be placed in the openings $a b c d$, so as to touch it-making twelve in all. If the pile be completed, we shall get a four-sided pyramid, of which each side is an equilateral triangle, as represented in Fig. 2. It will be seen. that, in these triangular faces, each ball (except, of course, those forming the edges) is touched by six others. 
Again, if we start with such a triangle, as in Fig. 3, where each ball is touched by six others, we can place one ball - the shaded one-so as to rest on three others, and can then place six more round it and touching it, as indicated by the dotted circles. In three of the triangular holes between the shaded ball and the dotted balls touching it we can place three more, so as to touch the sharled ball-again twelve touching it in all. If we complete

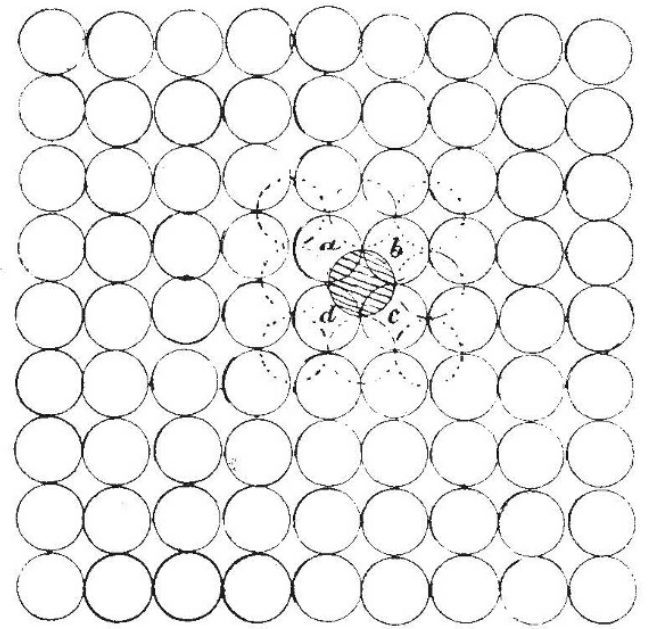

FIG. I.

the pile, we shall get the triangular pyramid represented by Fig. 4, where each of the three sides is a right-angled triangle, while the base is an equilateral triangle. It will be seen that in the faces of this pyramid each ball (except those outside) is touched by four others. In fact, the arrangement in these faces is the same as in the base of the former pyramid; and the two arrangements are really identical in the interior, only one has to be

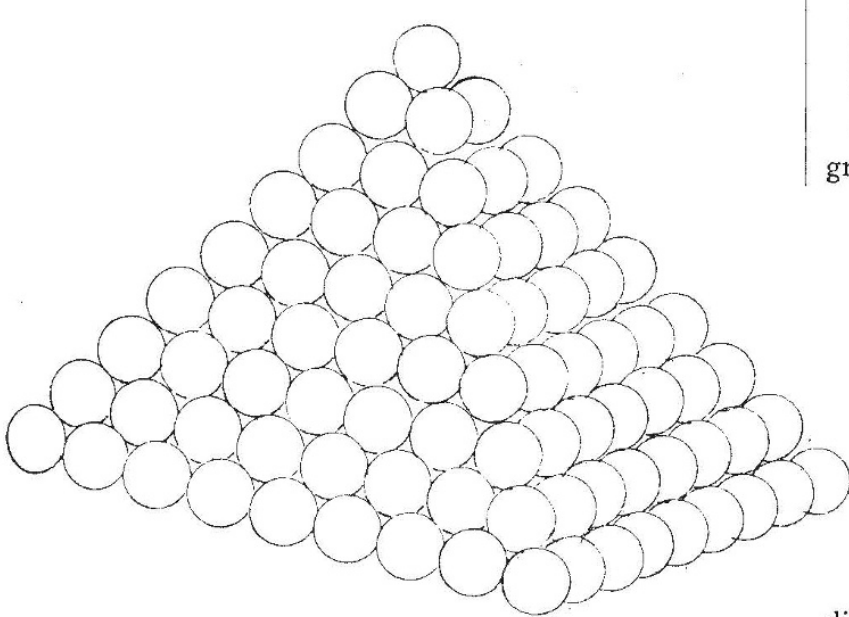

F1G. 2.

turned over in order to bring it into parallelism with the other. Fig. 2 represents half a regular octahedron; Fig. 4 the corner of a cube. Ellipsoids, if they are all equal and similar to one another, can be packed in precisely the same way, so that each is touched by twelve others, provided their axes are kept parallel to each other - that is, if they are all oriented alike. This, then, by the laws of energy, will be the arrangement which the mole- cules will assume, in consequence of mutual attraction, in passing from a fluid to a solid state.

Next, let us see how the packing of the molecules will affect the external form. And here I bring in the surfacetension. We are familiar with the effects of this force in the case of liquids, and if we adopt the usually received theory of it, we must have a surface-tension at the boundary of a solid, as well as at the surface of a liquid. I know of no actual measures of the surface-tension of solids; but Quincke has given us the surface-tensions of a number of substances at temperatures near their

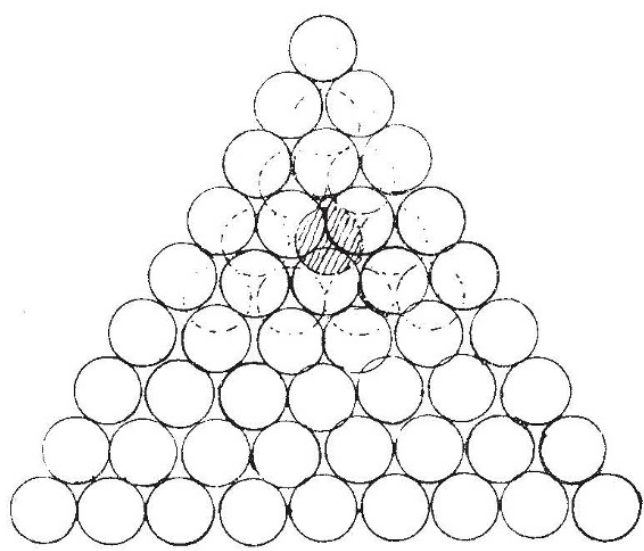

FIG. 3.

points of solidification, in dynes per lineal centimetre, as: follows :-

\begin{tabular}{llllr|lllll} 
Platinum & $\ldots$ & $\ldots$ & $\ldots$ & 1658 & Antimony & $\ldots$ & $\ldots$ & 244 \\
Gold $\ldots$ & $\ldots$ & $\ldots$ & $\ldots$ & 983 & Borax & $\ldots$ & $\ldots$ & $\ldots$ & 2 r 2 \\
Zinc $\ldots$ & $\ldots$ & $\ldots$ & $\ldots$ & 860 & Sodium carbonate & $\ldots$ & 206 \\
Tin $\ldots$ & $\ldots$ & $\ldots$ & $\ldots$ & $\ldots$ & 587 & Sodium chloride & $\ldots$ & Ir 4 \\
Mercury & $\ldots$ & $\ldots$ & $\ldots$ & 577 & Water & $\ldots$ & $\ldots$ & $\ldots$ & $86 \cdot 2$ \\
Lead $\ldots$ & $\ldots$ & $\ldots$ & $\ldots$ & 448 & Selenium $\ldots$ & $\ldots$ & $\ldots$ & $70 \cdot 4$ \\
Silver & $\ldots$ & $\ldots$ & $\ldots$ & 419 & Sulphur & $\ldots$ & $\ldots$ & $\ldots$ & $41 \cdot 3$ \\
Bismuth & $\ldots$ & $\ldots$ & $\ldots$ & 382 & Phosphorus & $\ldots$ & $\ldots$ & $41 \cdot 1$ \\
Potassium & $\ldots$ & $\ldots$ & $\ldots$ & 364 & Wax .. & $\ldots$ & $\ldots$ & $\ldots$ & $33 \cdot 4$ \\
Sodium & $\ldots$ & $\ldots$ & $\ldots$ & 253 & & & & &
\end{tabular}

The surface-tensions of most of the solids are probably greater than these, for the surface-tension generally

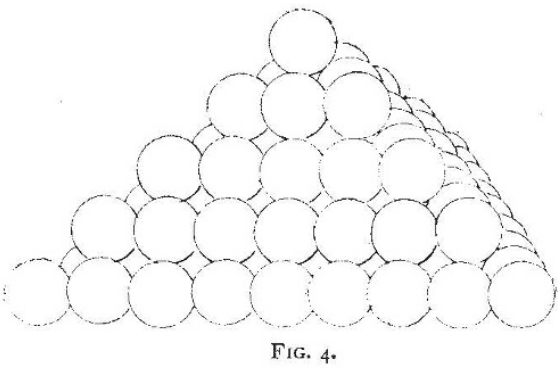

diminishes with increase of temperature; and you see that they amount to very considerable forces. We have to do, then, with an agency which we cannot neglect. In all these cases the tension measured is at a surface bounded by air, and is such as tends to contract the surface. We have, then, at the boundary between a crystallizing solid and the fluid, be it gas or liquid, out of which it is solidifying, a certain amount of potential energy ; and by the laws of energy the condition of equilibrium is, that this potential energy shall be a minimum. The accepted theory of surface-tension is that it arises from the mutual

NO. I I 29, VOL. 44] 
attraction of the molecules. The energy will therefore be a minimum for a surface in which the molecules are as closely set as possible.

Now, if you draw a surface through a heap of balls packed so that each is touched by twelve others, you will find that the surfaces which have the greatest number of centres of balls per unit area are all plane surfaces. That in which the concentration is greatest is the surface of a regular octahedron, next comes that of a cube, then that of a rhombic dodecahedron, and so on according to the law of indices of crystallographers.

The relative numerical values of these concentrations are as follows, taking that of the faces of the cube as unity :-

\begin{tabular}{llll|ll} 
Octahedron & $\ldots$ & $\ldots$ & $\mathbf{I} \cdot 1547$ & Tetrakishexahedron & $0 \cdot 4472$ \\
Cube $\ldots$ & $\ldots$ & $\ldots$ & $\mathrm{I} \cdot 0000$ & Eikositessarahedron & $0 \cdot 4083$ \\
Dodecahedron & $\ldots$ & $0 \cdot 707 \mathrm{I}$ & Triakisoctahedron & $\ldots$ & 0 \\
Dod333
\end{tabular}

We do not know that the surface-tension is exactly in the inverse proportion to the concentration, all that we can at present say is that it increases as the concentration diminishes.

If, then, the molecules occupy spherical spaces, the bounding surface will tend to be a regular octahedron.

But we have another point to consider. If a solid is bounded by plane surfaces, there must be edges where these planes meet. At such an edge the surface-tensions will have a resultant (see Fig. 5) tending to compress the mass, which must be met by a corresponding opposite pressure, and unless there is some internal strain there must be a corresponding resultant of the tensions on the opposite side of the crystal. Hence, if one face of a form

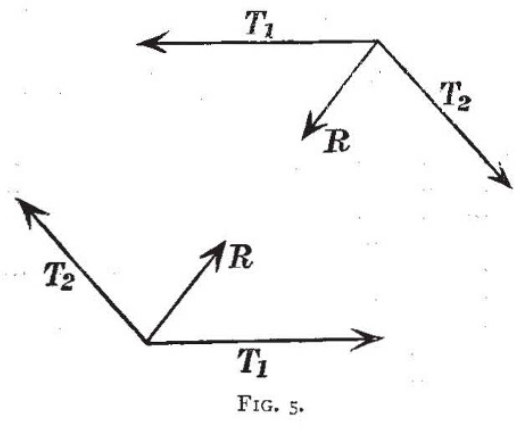

is developed the opposite face will also be developed; and generally, if one face of a form be developed all the faces will be developed; and if one edge, or angle, be truncated, all the corresponding edges, or angles, will be truncated. Were it otherwise, there would not be a balance between the surface-tensions in the several faces. But there is another point to be taken into account. The surface energy may become less in two ways-either by reducing the tension per unit surface, or by reducing the total surface. When a liquid separates from another fluid, as chloroform from a solution of chloral hydrate on adding an alkali, or a cloud from moist air, the liquid assumes the form which, for a given mass, has the least surfacethat is, the drops are spherical. If you cut off the projecting corners and plane away the projecting edges of a cube or an octahedron, you bring it nearer to a sphere, and if you suppose the volume to remain constant, you still diminish the surface. And if the diminution of the total surface is not compensated by the increased energy on the truncations, there will be a tendency for the crystals to grow with such truncations. The like will be true in more complicated combinations. There will be a tendency for such combinations to form, provided the surface energy of the new faces is not too great as compared with that of the first simple form.

But it does not always happen that an octahedron of alum develops truncated angles. This leads to another point. To produce a surface in a continuous mass requires a supply of energy, and to generate a surface in the interior of any fluid is not easy. Air may be supersaturated with aqueous vapour, or a solution with a salt, and no cloud or crystals be formed, unless there is some discontinuity in the mass, specks of dust, or something of the kind. In like manner, if we have a surface already, as when a supersaturated solution meets the air or the sides of the vessel containing it, and if the energy of either of these surfaces is less than that of a crystal of the salt, some energy will have to be supplied in order to produce the new surface, but not so much as if there were no surface there to begin with. Hence, crystals usually form on the sides of the vessel or at the top of the liquid. When a solid separates from a solution there is generally some energy available from the change of state, which supplies the energy for the new surface. But at first when the mass deposited is very small the energy available will be correspondingly small, and since the mass varies as the cube of the diameter of the solid, whereas the surface varies as the square of the diameter, the first separated mass is liable to be squeezed into liquid again by its own surface-tension. This explain s the usual phenomena of supersaturated solutions. A deposit occurs most easily on a surface of the same energy as that of the deposit, because the additional energy required is only for the increased extent of surface. It explains, too, the tendency of large crystals to grow more rapidly than small ones, because the ratio of the increase of surface to that of volume diminishes as the crystal grows.

While speaking of the difficulty of creating a new surface in the interior of a mass, the question of cleavage suggests itself. In dividing a crystal we create two new surfaces-one on each piece, and each with its own energy. The division must therefore take place most readily when that surface energy is a minimum. Hence the principal cleavage of a crystal made up of molecules having their motions comprised within spherical spaces will be octahedral. As a fact, we find that the greater part of substances which crystallize in the octahedral, or regular system, have octahedral cleavage. But not all ; there are some, like rock salt and galena, which cleave into cubes, and a very few, like blende, have their easiest cleavage dodecahedral. These I have to explain. I may, however, first observe that some substances-as, for instance, fluor-spar - which have a very distinct octahedral cleavage are rarely met with in the form of octahedra, but usually in cubes. In regard to this, we must remember that the surface energy depends upon the nature of both the substances in contact at the surface, as well as on their electrical condition, their temperature, and other circumstances. The closeness of the molecules in the surface of the solid determines the energy, so far as the solid alone is concerned; but that is not the only, though it may be the most important factor conducing to the result. It is therefore quite possible that, under the circumstances in which the natural crystals of fluor were formed, the surface energy of the cubical faces was less than that of the octahedral, although when we experiment on them in the air it is the other way. This supposition is confirmed by the well-known fact that the form assumed by many salts in crystallizing is affected by the character of the solution. Thus alum, which from a solution in pure water always assumes the octahedral form, takes the cubic form when the solution has been neutralized with potash.

To return to the cubic and dodecahedral cleavages. If we suppose the excursions of the parts of the molecule to be greater in one direction than in the others, the figure within which the molecule is comprised will be a prolate spheroid ; if less, an oblate spheroid. Now, as already explained, the spheroids will be packed as closely as possible if the axes are all parallel and each is touched

NO. I I 29 , VOL. 44] 
by twelve others. Now suppose the spheroids arranged as in Fig. 6, with their axes perpendicular to the plane of the figure; place the next layer in the black triangular spaces, and complete the pyramid. The three faces of the pyramid will be equal isosceles triangles; and if the spheroids be oblate, and the axis half the greatest diameter, the three angles at the apex of the pyramid will be right angles. The crystal will have cubic symmetry, but the relative condensation in the faces of the cube, octahedron, and dodecahedron, will be as $\mathrm{I}: 0^{\circ} 5774: 0.707 \mathrm{~J}$. The easiest cleavage would therefore be cubic, as in rock salt and galena.

Again, if the spheroids have their axes and greatest diameters in the ratio of $I: \sqrt{2}$, and we place four, as in Fig. 7, with their axes perpendicular to the plane of the figure, then place one upon them in the middle, and then four more upon it, in positions corresponding to those of the first four, we get a cubical arrangement, the centre of

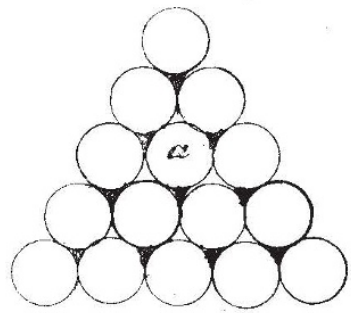

Fig. 6.

a spheroid in each angle of a cube, and one in the centre of the cube. Crystals so formed will have cubic symmetry, but the concentration of molecules will be greatest in the faces of the dodecahedron, and their easiest cleavage will be, like that of blende, dodecahedral.

If spheroids of any other dimensions be arranged, as in Figs. I and 2, with their axes perpendicular to the plane of Fig. I, we shall get a crystal with the symmetry of the pyramidal system. If the spheroids be prolate, the fundamental octahedron will be elongated in the direction of the axis, and if sufficiently elongated, the greatest condensation will be in planes perpendicular to the axis, and the easiest cleavage, as in prussiate of potash, in those planes. On the other hand, if the spheroids be sufficiently oblate, the easiest cleavage will be parallel to the axis.

If spheroids be arranged, as in Fig. 6, with their axes

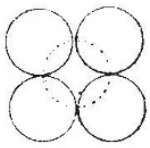

FIG. 7.

perpendicular to the plane of the figure, they will, in general, produce rhombohedral symmetry, with the rhombs acute or obtuse, according to the length or shortness of the axes of the spheroids. The cubical form already described is only a particular case of the rhombohedral. If the ratio between the axes of the spheroids and their greatest diameters be only a little greater, or a little less, than $I: 2$, the condensation will be greatest in the faces of the rhombohedron, and the easiest cleavage will be rhombohedral, as in calcite. If the spheroids be prolate, the easiest cleavage will be perpendicular to the axis of symmetry, as in beryl and many other crystals. Such crystals have a tendency to assume hexagonal forms-equiangular six-sided prisms and pyramids. To explain this, it may be seen in Fig. 6 that, in placing the next layer upon the spheroids represented in the figure, the three spheroids which touch that marked a may

NO. I I 29 , VOL. 44] occupy either the three adjacent white triangles or the three black ones. Either position is equally probable. The layer occupying the white triangles is in the position of a twin to that occupying the black triangles. So far as the central parts of the layer are concerned, it will make no difference in which of these ways the molecules are packed. It is only at the edges that the surfacetension will be affected. If the form growing be a rhombohedron, a succession of alternating twins will produce a series of alternating ridges and furrows in the rhombohedral faces, which will give rise to increased surface-tension, which will tend to prevent the twinning. On the other hand, an hexagonal form and its twin, formed in the way indicated, are identical, and we have in this fact a cause tending to the production of hexagonal forms. This tendency is increased by the fact that, for a given volume, the total surface of the hexagonal forms is in general less than that of the rhombohedral. Indeed, such forms lend themselves to the formation of almost globular crystals, as is well seen in pyromorphite and mimetite.

If the spheroids be arranged with their axes in other positions than those we have been discussing, or if the molecules occupy ellipsoidal spaces, they will, when packed so that each is touched by twelve others, give figures of less symmetry. The results may be worked out on the lines indicated in the foregoing discussion, and will be found to correspond throughout to the observed facts.

Bravais long ago proposed various arrangements of molecules to account for crystalline forms, and Sohncke has extended them to further degrees of complication in order to account for additional facts in crystallography But neither of them has given any reason why the molecules should assume such arrangements. To me it seems that only one arrangement can be spontaneously assumed by the molecules, and that the varieties of crystalline form depend on the dimensions of the ellipsoids and the orientation of their axes. Curie also has indicated that the development of combined forms, as those of cube and octahedron, will depend on the surface-tensions in the faces of these forms, but he has not indicated how the surface-tension is connected with the crystalline arrangement, or why the energy of a cubic face should be greater or less than that of an octahedral face.

We are now in a position to understand the interesting facts brought forward by Prof. Judd in a discourse delivered at the Royal Institution early this year. However long a crystal has been out of the solution, or vapour, from which it was formed, its surface-tension will remain unaltered, and when it is replaced it will grow exactly as if it had not been removed. Also, if any part be broken off it, the tension of the broken surface will, if it be not a cleavage face, be greater than on a face of the crystal, and in growing, the laws of energy necessarily cause it to grow in such a way as to reduce the potential energythat is, to replace the broken surface by the regular planes of less surface energy. The formation of "negative crystals" by fusing a portion in the interior of a crystalline mass, is due to the same principle. Surfaces of least energy will be most easily produced inside as well as outside, and in a crystalline mass of course they will be parallel to the external faces of the crystal. We see the same thing in the action of solvents. Most metals assume a crystalline texture on cooling from fusion, and when slowly acted on by dilute acids the surfaces of greater energy are most easily attacked, in accordance with the laws of energy, and the undissolved metal is left with surfaces of least energy which are the faces of crystals. This is easily seen on treating a piece of tin plate, or of galvanized iron, with very dilute aqua regia. In fact, solution is closely connected with surface energy. It is probably the low surface energy of one form of crystals of sulphur which makes them insoluble in carbon 
disulphide, and this low surface energy may be an electrical effect.

I pointed out that the development of all the faces of a form, and the similar modification of all corresponding edges and angles of a crystal, is in general necessary in order to produce equilibrium under the surface-tensions. But we sometimes find crystals with only half the modifications required for symmetry. In such cases the surfacetensions must produce a stress in the interior tending to deform the molecules. When the crystal was growing, there must have been equilibrium, and therefore a pressure equal and opposite to this effect of the surfacetension. There are various ways in which we may suppose that such a force would arise. The electric field might give rise to a stress in opposition to the aggregation of the molecules in the closest possible way, and then the crystal would grow such faces as would produce an equal and opposite stress. Inequalities of temperature, or the presence of molecules of other kinds amongst those of the crystal, might produce similar results. When the stress due to electricity, or to temperature, was removed by change of circumstances, that due to the surface-tensions would persist, and the crystal would be left with an internal strain. Crystals of this sort, with unsymmetric faces, generally betray the internal strain, either by developing electricity of opposite kinds at the two ends when heated or cooled, or they affect polarized light, rotating the plane of polarization. That these effects are due to the internal strain is shown by the fact that tourmalines, and other crystals, which are pyro electric when unsymmetrical, show no such property when symmetrically grown. Also sodium chlorate in solution, quartz when fused, and so on, lose their rotatory power. Substances which in solution show rotatory power, as a rule develop unsymmetric crystals. This is well seen in the tartrates. The constitution of the molecules must be such that they will not, without some strain, form crystals ; and equilibrium, when the crystal is growing, is attained by means of the opposing stress due to want of symmetry in the surface-tensions. In all such crystals the rotatory power of the solution disappears in whole or in part. We cannot test this in biaxial crystals, but, according to Des Cloiseaux, sulphate of strychnine is the only substance which shows rotation both in the solution and in the crystalline form, and in it the rotatory power is much increased by the crystallization. Effects comparable with these may be produced by mechanical means. A cube of rock salt, which has no effect on plane-polarized light in its ordinary state, changes the plane of polarization when it is compressed in a vice. And a cleavage slice of prussiate of potash, which is uniaxial, may by compression be distorted so as to give in a convergent beam of polarized light elliptical rings, and two eyes like a biaxial crystal.

\section{THE ERUPTION OF VESUVIUS OF JUNE 7,} I891.

URING the latter part of 1890 and the early part of the present year, the central activity of Vesuvius has very slightly varied, except about the new year, when it was considerably increased, rising to the third or fourth degree, simultaneous with the stoppage of the lateral outflow of lava that had been going on since August 7 I 890 . Since then, up to the present outburst, the central activity has been generally at the first degree, and the cone of eruption has slowly grown in height.

On June I there was a crater within the central eruptive cone, of about $50 \mathrm{~m}$. in diameter, near the centre of which was the eruptive vent, surrounded by another embryonic eruptive cone. On that day, four small eruptive mouths opened around the embryonic cone in the bottom of the central crater, the smallest being to the east.

Thus the volcano remained till June 7 , at io a.m., when activity stopped, only a small quantity of vapour escaping from central vents. At midday a radial cleft opened at the north toe of the cone of eruption (May I88y, June I89I) traversing towards its east end, the little sickleshaped ridge, the remnant of the I $885-86$ crater, but, as yet, gave out little vapour. At 4 to 4.30 p.m., shocks of earthquake commenced, limited only to the upper slopes of Vesuvius, and simultaneous with the extension of the radial fissure down the side of the great Vesuvian cone for nearly half its way opposite the Punta del Nasone of Monte Somma, from which, at about 5.30 p.m., issued a little lava, whilst from the upper extremity of the fissure at the toe of the cone of eruption much vapour escaped, so that from Naples the smoke-plume arose from this point. From 5.30 to 7 p.m. the fissure still extended lower, accompanied from time to time by local earthquakes, noises, and the elevation of columns of black dusty smoke. At a few minutes to 7 the floor of the Atrio del Cavallo was reached, and a remarkably black column of smoke had arisen.

My friend Dr. L. Sambon saw this column arise, and came to inform me immediately, as I had left off watching the mountain at 5.30. After taking a photo of the mountain, we left Naples at 9 p.m., spent some time in inquiries at Resina and near the Observatory. Everything was now dark, as the volcano had calmed down at 8 p.m. At 2 a.m. June 8 , we were at the eastern extremity of the Observatory ridge, and commenced to wend our way across the lava surface towards Monte Somma. We were at the lowest part of the depression at the west end of the Atrio del Cavallo, where it joins the Fossa della Vetrana, and along which some of the largest lava-streams have flowed (1855, 1872, \&c.) when suddenly on our right above us $(2.23 \mathrm{a}$.m. ) a vast quantity of bright red vapour arose from the new outpour of lava. We hastened our steps as much as the road and our lantern would allow us, so as to reach the escarpment of Monte Somma, the foot of which was followed till near the Punta del Nasone, and close to the theatre of eruption. Here we clambered up some distance above the level of the Atrio to watch events whilst we ate our late supper or early breakfast. Along the slope of the great cone in the line of fissure were a few luminous points from some pieces of still uncooled lava of the little that had oozed forth from the lower half of the fissure. At about 60 or 80 yards from the foot of the great cone two or three fountains of lava were throwing up jets of molten rock for 2 or $3 \mathrm{~m}$., and the lava was slowly spreading out on the almost horizontal plain of the Atrio in several tongues. The lava must have still been high in the main chimney, as the vapour that issued at the top of the fissure showed a slightly red illumination. So we remained till daylight, when we could see the fissure on the side of the cone. The mouth that formed at 5.30 the previous day was still smoking a little, whilst the fissure below it sent off several ramifications at an acute angle like the branches of an inverted tree, from several of which little streams of lava had been given out, where they had soon consolidated. We now followed the base of the great cone to the lower railway station, where we found all the people up and dressed, frightened by the strong shock and noises at $2.23 \mathrm{a} . \mathrm{m}$., coincident with the fresh outflow of lava that we had witnessed, but which shocks we had not felt, although they were described as the strongest that had been felt.

Having ascended to the summit of Vesuvius, we found the central crater rapidly enlarging by the falling in of its edges. From the new fissure at its summit was issuing much vapour under pressure, and rich in sulphurous acid, which is, even in traces, intolerable ; and the hot air coming from innumerable new fissures rendered approach very difficult. We did, in fact, once jump across part of the fissure, but returned much quicker on account of the hot irritant vapours. An approach from the opposite

NO. I I 29, vOL. 44] 